\title{
SPN Gene
}

National Cancer Institute

\section{Source}

National Cancer Institute. SPN Gene. NCI Thesaurus. Code C101151.

This gene is involved in leukocyte migration. 